\title{
Variabilidade espacial de variáveis físico-hídricas do solo em um pomar de lima ácida Tahiti, irrigado por microaspersão
}

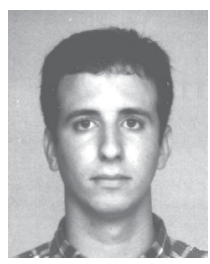

\author{
Maurício A. Coelho Filho ${ }^{2}$, Rubens D. Coelho ${ }^{3}$ \& Antônio C.A. Gonçalves ${ }^{4}$
}

${ }^{1}$ Parte do trabalho de Dissertação de Mestrado apresentada pelo primeiro autor ao Curso de Pós-Graduação em Irrigação e Drenagem da ESALQ/USP

2 ESALQ/USP, CP 9, CEP 13418-900, Piracicaba, SP. E-mail: macoelho@carpa.ciagri.usp.br (Foto)

${ }^{3}$ ESALQ/USP. E-mail: rdcoelho@carpa.ciagri.usp.br

4 UEM. Maringá, PR. E-mail: acagoncalves@uem.br

Protocolo $130-23 / 10 / 2000$

\begin{abstract}
Resumo: Este trabalho foi desenvolvido em um pomar de lima ácida Tahiti, irrigado por microaspersão, e teve como objetivo determinar a variabilidade espacial de variáveis do solo, utilizadas no manejo da irrigação. Amostras de solo foram coletadas a $0,20 \mathrm{~m}$ de profundidade, em pontos de amostragem definidos segundo uma malha com 126 amostras para determinação dos teores de areia, silte e argila, densidade do solo (Ds) e da água disponível (AD). O limite superior da faixa de água disponível foi a umidade na capacidade de campo $\left(U_{c c}\right)$. O limite inferior foi tomado como a umidade no limite de funcionamento do tensiômetro $\left(U_{\mathrm{ft}}\right)$ e na umidade no ponto de murcha permanente $\left(U_{\mathrm{pmp}}\right)$. O coeficiente de variação $(\mathrm{CV})$ foi baixo para as variáveis $\mathrm{U}_{c c}, \mathrm{U}_{\mathrm{ft}}, \mathrm{U}_{\mathrm{pmp}}$ e argila, com valores de $\mathrm{CV}$ crescentes nesta ordem. Areia, silte, $A D_{\mathrm{pmp}}$ e $A D_{\mathrm{ft}}$, apresentaram média variação. As variáveis manifestaram estrutura de dependência espacial, modelada por meio de semivariogramas, os quais permitiram a interpolação por "krigagem". A distribuição espacial da umidade foi semelhante à da argila, indicando forte correlação espacial, confirmada pelos semivariogramas cruzados.
\end{abstract}

Palavras-chave: variabilidade espacial, água disponível, geoestatística, citros

\section{Spatial variability of soil hydrophysical variables in a Tahiti lime orchard irrigated by micro-sprinkler}

\begin{abstract}
This study was carried out in a Tahiti lime orchard, in a micro-sprinkler irrigated area, aiming to evaluate the soil hydrophysical characteristic variability related with irrigation management. Soil samples were collected at $0.20 \mathrm{~m}$ depth, based on a grid with 126 samples to determine sand, silt and clay contents, bulk density and available water (AW). The upper limit of available water was calculated by values of field capacity $\left(W_{f c}\right)$ and the lower limit by tensiometer working range $\left(\mathrm{W}_{\mathrm{t}}\right)$ and permanent wilting point $\left(\mathrm{W}_{\mathrm{pwp}}\right)$. Low variation was observed for $\mathrm{W}_{\mathrm{fc}}, \mathrm{W}_{\mathrm{t}^{\prime}}, \mathrm{W}_{\mathrm{pwp}}$ and clay content, with the variation coefficient increasing in this order. The results showed medium variability for sand, silt, $\mathrm{AW}_{\mathrm{t}}$ and $\mathrm{AW}_{\mathrm{pwp}}$. In the scope of the present work, the spatial independence was verified. By using the semivariograms, it was possible to interpolate by kriging. The spatial distribution of soil water $\left(\mathrm{W}_{\mathrm{fc}}, \mathrm{W}_{\mathrm{t}}\right.$ and $\left.\mathrm{W}_{\mathrm{pwp}}\right)$ was similar to the clay content. The spatial correlation between these variables was described by structured cross-semivariograms.
\end{abstract}

Key words: spatial variability, available water, geostatistics, citrus

\section{INTRODUÇÃO}

Desde o início do século XX, a variabilidade do solo tem preocupado pesquisadores por proporcionar diferenças no desenvolvimento e na produtividade das culturas, alterando resultados de pesquisas, mesmo com o solo considerado homogêneo em parcelas experimentais. Problemas como esses ocorrem devido à subjetividade ao se traçar os delineamentos sem um conhecimento prévio da estrutura espacial de variáveis do solo. Segundo Vieira \& Maria (1995) em razão das variáveis não serem homogêneas e apresentarem dependência espacial, a variabilidade causa problemas em experimentos de campo, especialmente nos que apresentam parcelas experimentais grandes. A caracterização da variabilidade espacial é essencial para um entendimento melhor das inter-relações entre propriedades do solo e fatores ambientais. Com um modelo de dependência espacial de variáveis do solo e planta, a geoestatística possibilita a estimativa em pontos não amostrados, viabilizando o mapeamento da variável. Este procedimento pode auxiliar na 
melhor distribuição das parcelas e dos blocos experimentais no campo, assim como o manejo mais racional da água, de fertilizantes e de defensivos agrícolas.

Existem muitas causas de variação nas características dos solos, relacionadas com os fatores de formação, com o manejo agronômico e com a erosão (Castrignano \& Stelluti, 1999). Como resultado, a natureza da variabilidade espacial do solo estudado depende da escala de observação e da variável em questão. Segundo Tragmar et al. (1985) propriedades do solo muito afetadas pelo manejo apresentam maior variabilidade que as ligadas às características morfológicas (cor e horizontes), físicas (tamanho das partículas e densidade das partículas) e químicas (pH). Oliveira (1973) estudando um Latossolo Roxo e um Latossolo Vermelho-Escuro-Orto, verificou que as características físicas e morfológicas do solo apresentam maior homogeneidade que as químicas e encontrou, para a água retida a $1 / 3$ atm e a 15 atm, argila e $\mathrm{pH}$, coeficientes de variação inferiores a 10\%. Por outro lado, cálcio, magnésio, potássio e soma de bases, apresentaram coeficientes acima de 40\%. Resultados semelhantes foram encontrados por Paz et al. (1996) em que os parâmetros físico-químicos apresentaram menor variabilidade que os macro e micronutrientes do solo. Souza et al. (1997) avaliando propriedades físicas e químicas de um Latossolo Amarelo distrófico em pomar cítrico em que as amostras foram coletadas na zona adubada sob as copas das árvores, verificaram maior variabilidade para o fósforo e o potássio. Moraes et al. (1993) trabalhando com Terra Roxa estruturada, verificaram que os coeficientes de variação para a umidade do solo nas tensões estudadas, apresentaram valores inferiores a $10 \%$.

Dentre as muitas variáveis que interferem no crescimento vegetal, a água pode ser considerada a principal, sendo muito importante sua manutenção em níveis não limitantes ao longo do ciclo produtivo da cultura, seja através de técnicas de conservação da água no solo ou pelo fornecimento de água por irrigação. A disponibilidade de água no solo é afetada por sua textura e estrutura, esta última sensível ao manejo agronômico da área, o que afeta, conseqüentemente, a variabilidade da capacidade de retenção de água no solo.

$\mathrm{Na}$ experimentação, assim como na elaboração e manejo de projetos de irrigação, é fundamental o conhecimento da água disponível. Muitas vezes, seja pelas dificuldades no acesso aos laboratórios que realizam análises, custo e até negligência, não se realizam amostragens adequadas para uma caracterização mais detalhada da área irrigada, resultando em insucesso associado a uma escolha inadequada do sistema de irrigação, turno de rega, lâmina de água, entre outros. Dificuldades como estas favorecem a intensa simplificação do complexo solo e, conseqüentemente, da planta, que tem sua atividade radicular afetada por modificações nas propriedades físico-hídricas do solo. Ao se assumir que uma extensa área apresenta suposta homogeneidade, conseqüências graves podem ocorrer, relacionadas à contaminação de águas subterrâneas, salinização, degradação do solo, má utilização de recursos hídricos e de energia, além de menor retorno econômico.

O presente trabalho teve como objetivo avaliar e descrever a distribuição espacial de variáveis físico-hídricas do solo, importantes para o manejo da irrigação, em pomar de lima ácida
'Tahiti', como subsídio para um manejo racional de irrigação e experimentação, na área estudada.

\section{MATERIAL E MÉTODOS}

\section{Coleta e análise laboratorial}

O presente trabalho foi desenvolvido na Área Experimental de Irrigação (Fazenda Areão) do Departamento de Engenharia Rural, Escola Superior de Agricultura "Luiz de Queiroz", Universidade de São Paulo. As coordenadas geográficas da área experimental são: $22^{\circ} 42^{\prime} 30^{\prime \prime}$ de latitude Sul e 47 $30^{\circ} 00^{\prime \prime} d e$ longitude Oeste, estando a $576 \mathrm{~m}$ acima do nível do mar. O clima da região, segundo classificação de Köeppen, é do tipo Cwa, com médias de precipitações anuais de $1.253 \mathrm{~mm}$, temperatura média anual de $21,2^{\circ} \mathrm{C}$, umidade relativa de $74 \%$, velocidade do vento: $2,2 \mathrm{~m} \mathrm{~s}^{-1}$ com direção E/SE predominante. O período mais seco do ano concentra-se nos meses de junho, julho e agosto. O solo é classificado como Terra Roxa Estruturada (Alfisol), série Luiz de Queiroz. A área total de estudo foi de $5.975 \mathrm{~m}^{2}$, com declividade média de 8,5\%.

Os estudos foram realizados em um pomar de lima ácida 'Tahiti' enxertada em limoeiro 'Cravo', com idade de 5 anos. Do total de plantas do pomar, foram selecionadas 112, onde foi instalado o experimento. A área utilizada constou de sete fileiras de plantas, sendo o espaçamento entre plantas de $8 \times 7 \mathrm{~m}$. Somente entre duas fileiras de plantas o espaçamento é de $9 \times 7 \mathrm{~m}$, em função da existência de um terraço.

Para o estudo da granulometria do solo (teor de argila, silte e areia) foram coletadas 126 amostras deformadas, formando uma malha de pontos distribuídos no pomar (Figura 1). As amostras foram coletadas a uma profundidade de $0,2 \mathrm{~m}$ e $1,2 \mathrm{~m}$ distante do tronco das plantas.

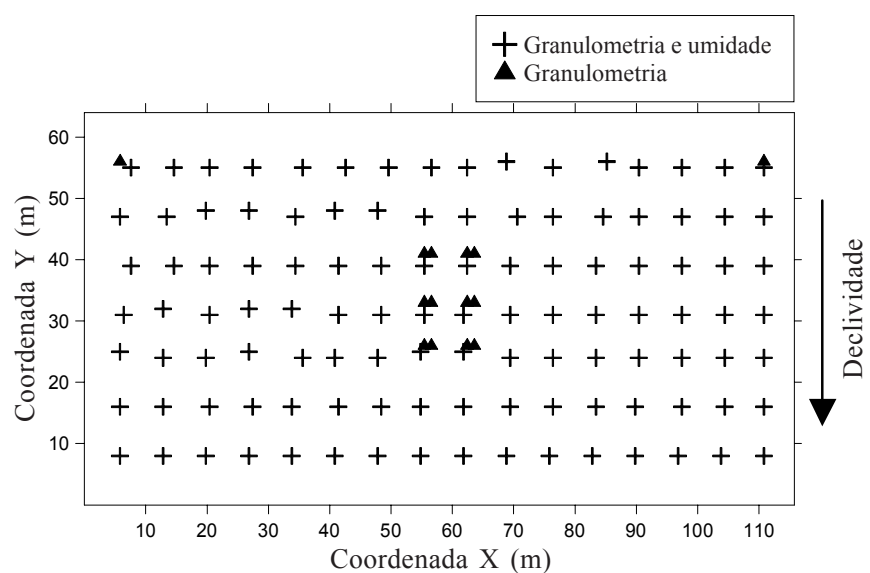

Figura 1. Esquema de amostragem realizada na área experimental, destinada ao estudo granulométrico e de umidade do solo

As amostras destinadas à elaboração das curvas de retenção, foram coletadas nas mesmas trincheiras utilizadas no estudo de granulometria, em anel com volume conhecido. Por outro lado, a retenção de água do solo foi determinada no Laboratório de Solos do Departamento de Engenharia Rural da ESALQ/USP. Foi avaliada a umidade do solo nas tensões de $10 \mathrm{kPa}$ (adotada como capacidade de campo - $\mathrm{U}_{\mathrm{cc}}$ ), $80 \mathrm{kPa}$ (tensão correspondente ao limite de funcionamento do 
tensiômetro - $\mathrm{U}_{\mathrm{ft}}$ ) e $1500 \mathrm{kPa}$ (tensão no ponto de murcha permanente - $\mathrm{U}_{\mathrm{pmp}}$ ).

A disponibilidade de água no solo (AD) foi calculada pela seguinte equação:

$$
\mathrm{AD}(\%)=\left(\mathrm{U}_{\mathrm{cc}}-\mathrm{U}_{\mathrm{cri}}\right) 100
$$

em que: $\mathrm{U}_{\text {cri }}$ - umidade na tensão correspondente ao limite de funcionamento do tensiômetro $\left(\mathrm{U}_{\mathrm{ft}}\right)$ e ao ponto de murcha permanente $\left(\mathrm{U}_{\mathrm{pmp}}\right)$.

\section{Análise estatística}

Foram realizadas a análise descritiva e a exploratória das variáveis, a verificação da dependência espacial e a interpolação, pelo método de "krigagem". Para uma primeira análise de cada propriedade estudada, construiu-se um gráfico da distribuição dos dados no campo, "post plot". Este procedimento foi importante para o conhecimento preliminar da distribuição na área, além de se verificar a possível existência de tendência dos valores, negando a estacionaridade intrínseca. Para tanto, os resultados obtidos para cada variável, foram separados em 4 intervalos de classes, com igual quantidade de dados (Libardi et al, 1996). Verificado algum problema de tendência no comportamento dos dados, foi realizado o processo de remoção mediante o refinamento pela mediana, conforme Hamlett et al. (1986). Neste caso, o conjunto de dados utilizado no estudo de dependência espacial passou a ser os resíduos do refinamento.

Assumindo-se a estacionaridade intrínseca, a continuidade espacial foi estudada construindo-se semivariogramas (McBratney \& Webster, 1986). A função semivariância foi calculada pela Eq. 2 (Journel \& Huijbregts, 1978):

$$
\hat{\gamma}(h)=\frac{1}{2 N(h)} \sum_{i=1}^{N(h)}\left[Z\left(s_{i}\right)-Z\left(s_{i}+h\right)\right]^{2}
$$

em que: $\mathrm{N}(\mathrm{h})$ - número de pares de valores medidos $\mathrm{Z}\left(\mathrm{s}_{\mathrm{i}}\right), \mathrm{Z}\left(\mathrm{s}_{\mathrm{i}}+\mathrm{h}\right)$ separados pelo vetor $h$. Para variáveis com dependência espacial, espera-se que o semivariograma comece com um baixo valor, denominado "nugget effect" $\left(\mathrm{C}_{\mathrm{o}}\right)$ e seja crescente com a distância, até um valor denominado alcance, correspondente ao limite da dependência espacial. A partir do alcance, a variável apresenta-se espacialmente independente e a semivariância tende a estabilizar-se em torno de um valor denominado patamar $\left(\mathrm{C}+\mathrm{C}_{\mathrm{o}}\right)$, em que C é o "sill" ou componente estrutural. Para cada semivariograma foram ajustados os modelos exponencial, esférico e gaussiano. A decisão pelo melhor modelo e respectivos coeficientes foi tomada com base na validação cruzada.

A "krigagem" é essencialmente uma média móvel ponderada (Vauclin et al. 1983) em que a estimativa $Z^{*}$, associada à posição $\mathrm{S}_{\mathrm{o}}$, pode ser obtida pela equação:

$$
\mathrm{Z} *\left(\mathrm{~s}_{\mathrm{o}}\right)=\sum_{\mathrm{i}=1}^{\mathrm{n}} \lambda_{\mathrm{i}} \mathrm{Z}\left(\mathrm{s}_{\mathrm{i}}\right)
$$

sendo $\mathrm{Z}\left(\mathrm{S}_{\mathrm{i}}\right)$ o valor medido na posição $\mathrm{s}_{\mathrm{i} ;} \lambda_{\mathrm{i}}$ é o peso utilizado na "krigagem" que está associado ao valor medido i que ocupa a posição $\mathrm{s}_{\mathrm{i}} \mathrm{e} \mathrm{N}$ é o número de vizinhos medidos utilizados na estimativa. Os pesos atribuídos aos vizinhos são obtidos em função da variabilidade espacial da área, expressa pelo semivariograma experimental, obtido com os valores medidos. Enfim, quando se deseja comparar o padrão de variabilidade espacial de duas variáveis distintas, ou o padrão de uma mesma variável em diferentes regiões, o procedimento do escalonamento do semivariograma pode ser utilizado, facilitando a interpretação dos resultados. O escalonamento é baseado na seguinte expressão:

$$
\gamma_{\mathrm{esc}}=\frac{\gamma(\mathrm{h})}{\phi}
$$

onde $\phi$ corresponde ao fator de escalonamento, que pode ser a variância amostral ou outro fator que permita a padronização das escalas dos semivariogramas comparados (Gonçalves, 1997). O fator usado foi a variância amostral, conforme Vieira et al. (1997).

A correlação espacial entre argila e retenção da água no solo $\left(\mathrm{U}_{\mathrm{cc}} ; \mathrm{U}_{\mathrm{ft}} ; \mathrm{U}_{\mathrm{pmp}}\right)$ foi avaliada construindo-se semivariogramas cruzados, obtidos com a seguinte expressão:

$\gamma_{12}(h)=\frac{1}{2 N(h)} \sum_{i=1}^{N(h)}\left[z_{1}\left(x_{i}\right)-z_{1}\left(x_{i}+h\right)\right]\left[z_{2}\left(x_{i}\right)-z_{2}\left(x_{i}+h\right)\right](5)$

em que: $\mathrm{N}(\mathrm{h})$ - número de pares de valores $\left\{\left[\mathrm{z}_{1}\left(\mathrm{x}_{\mathrm{i}}\right), \mathrm{z}_{1}\left(\mathrm{x}_{\mathrm{i}}+\mathrm{h}\right)\right]\right.$, $\left.\left[\mathrm{z}_{2}\left(\mathrm{x}_{\mathrm{i}}\right), \mathrm{z}_{2}\left(\mathrm{x}_{\mathrm{i}}+\mathrm{h}\right)\right]\right\}$ separados por um vetor $\mathrm{h}$. Para os semivariogramas cruzados, o fator de escalonamento utilizado foi a covariância das variáveis analisadas.

\section{RESULTADOS E DISCUSSÃO}

\section{Textura e densidade do solo}

Os resultados referentes à análise descritiva para os teores de argila, silte, areia e densidade do solo (Ds) apresentados na Tabela 1 , indicaram ajuste à distribuição normal. Os valores de moda, mediana e média, para todas as variáveis, estão próximos, indicando distribuições simétricas, o que pode ser confirmado pelos valores de assimetria próximos de zero.

Tabela 1. Resumo estatístico do conteúdo de argila (\%), silte $(\%)$, areia (\%) e da densidade do solo Ds $\left(\mathrm{g} \mathrm{cm}^{-3}\right)$

\begin{tabular}{lcrrc} 
Parâmetros & Argila & Silte & Areia & \multicolumn{1}{c}{ Ds } \\
\hline $\mathrm{N}^{\mathrm{o}}$ de amostras & \multicolumn{1}{c}{126} & 126 & 126 & 112 \\
Média & 61,45 & 16,62 & 21,93 & 1,42 \\
Mediana & 62,35 & 16,68 & 21,38 & 1,42 \\
Mínimo & 44,31 & 7,94 & 11,16 & 1,24 \\
Máximo & 74,01 & 25,24 & 32,49 & 1,64 \\
Quartil inferior & 55,47 & 13,21 & 18,95 & 1,37 \\
Quartil superior & 67,46 & 19,73 & 25,32 & 1,47 \\
Amplitude & 29,71 & 17,30 & 21,33 & 0,40 \\
Desvio-Padrão & 7,17 & 4,33 & 4,38 & 0,08 \\
CV (\%) & 11,66 & 26,07 & 19,97 & 5,63 \\
Assimetria & $-0,29$ & 0,008 & 0,34 & 0,16 \\
Curtose & $-0,94$ & $-0,93$ & $-0,35$ & $-0,09$ \\
\hline
\end{tabular}


Analisando-se o gráfico de "box-plot" para o teor de argila, silte e areia e para densidade do solo, verifica-se elevada simetria para silte e densidade do solo (Figura 2). Quanto aos valores de argila, pode ser notada a ocorrência de uma dispersão maior dos dados para os valores abaixo do quartil inferior (assimetria negativa). Para areia, silte e densidade, a assimetria foi positiva (Tabela 1).
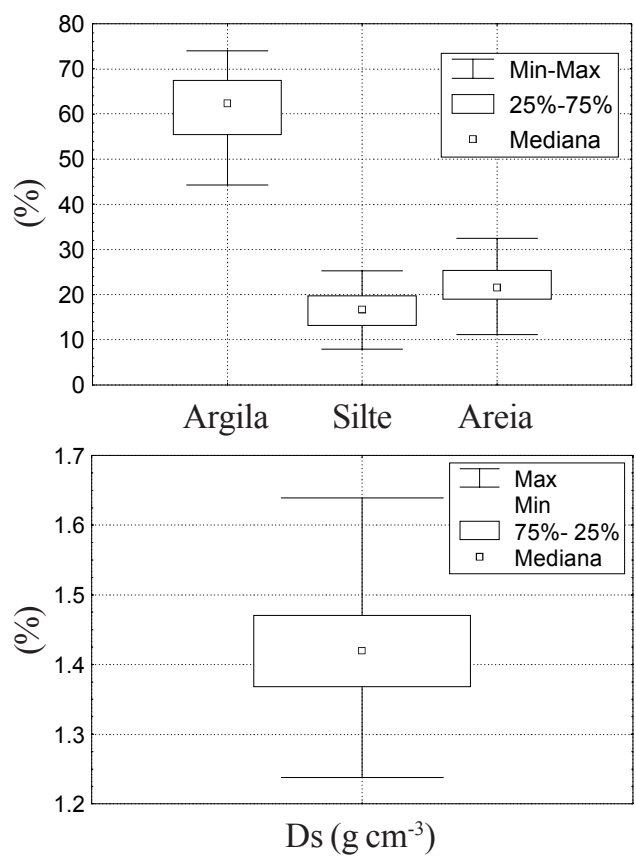

Figura 2. "Box-plot" para argila, silte, areia e densidade do solo

Os resultados referentes ao teste de Kolmogorov-Smirnov, indicaram normalidade dos dados para as variáveis estudadas. Para areia e argila, foram significativos em nível de 15 e 10\%, respectivamente, talvez refletindo o maior afastamento de zero para a assimetria.

O valor do coeficiente de variação para densidade do solo $(5,6 \%)$ indica pequena variação em torno da média, o que está de acordo com os resultados de Warrick \& Nielsen (1980) que classificaram esta propriedade como de baixa variação, não superando $10 \%$. Resultados semelhantes foram encontrados também por Moraes \& Libardi (1993) em Terra Roxa Estruturada Latossólica, trabalhando com amostragem em malha regular e coeficiente de variação na ordem de $5 \%$.

Os valores do coeficiente de variação $(\mathrm{CV})$ referentes à granulometria indicaram variabilidade baixa para argila $(11,6 \%)$ e média para silte e areia ( 20 e 26\%). Teor de argila apresentou maior amplitude total e interquartílica, quando comparado com as outras variáveis, o que justifica o baixo valor do CV. Os resultados da Tabela 1 revelaram que, em média, o solo é de textura argilosa.

Ao se analisar a distribuição espacial das variáveis pelo gráfico de "post-plot", verificou-se que as variáveis estudadas possuíram valores pertencentes à mesma classe, concentrados em determinadas regiões. Para argila, observou-se tendência de acréscimo nos teores em direção à coordenada $\mathrm{X}$, sendo a concentração desses valores maior na região inferior esquerda. Para areia, verificou-se a concentração de valores da mesma classe na região direita do gráfico e, para os valores pertencentes à classe superior, constatou-se sua concentração em grande parte no centro da área. Apesar da concentração das classes em determinadas regiões para argila, silte e areia, não se verificou tendência de variação de larga escala; nesse caso, assumiu-se a estacionaridade intrínseca. Para a densidade do solo, os valores altos se concentraram na parte superior da área experimental. $\mathrm{O}$ tráfego intenso de máquinas agrícolas pode ajudar a explicar esses valores. Na parte inferior da superfície, pode-se verificar a concentração de valores baixos para a densidade do solo. A clara tendência de decréscimo da densidade com a redução na coordenada $Y$, não permitiu assumir a estacionaridade intrínseca. O tratamento dos dados foi realizado para a remoção da tendência verificada nos dados originais, a qual não está presente nos resíduos que, por sua vez, foram assumidos com estacionaridade intrínseca (Figura 3). Todo o estudo de dependência espacial para densidade do solo foi realizado com os resíduos do refinamento, seguindo-se o mesmo padrão de análise realizado para os valores originais, na tentativa de se identificar pontos que pudessem ter afetado a distribuição.

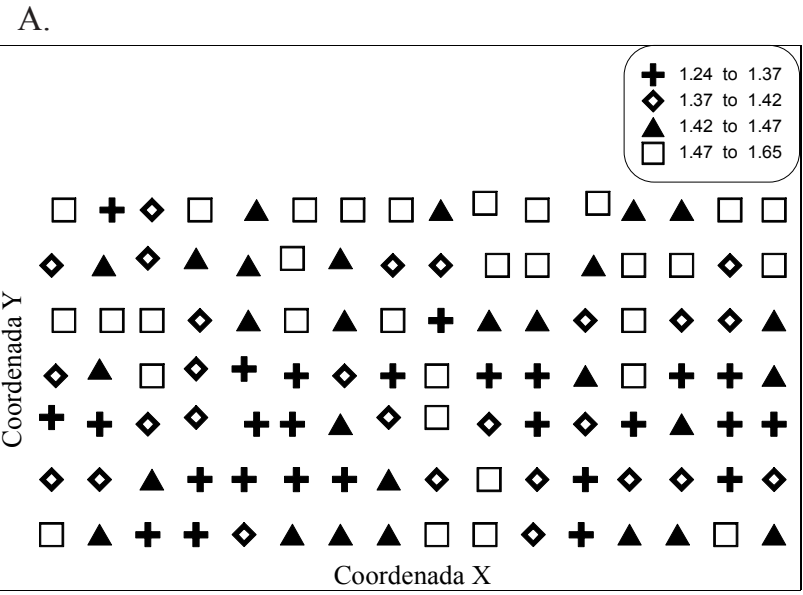

B.

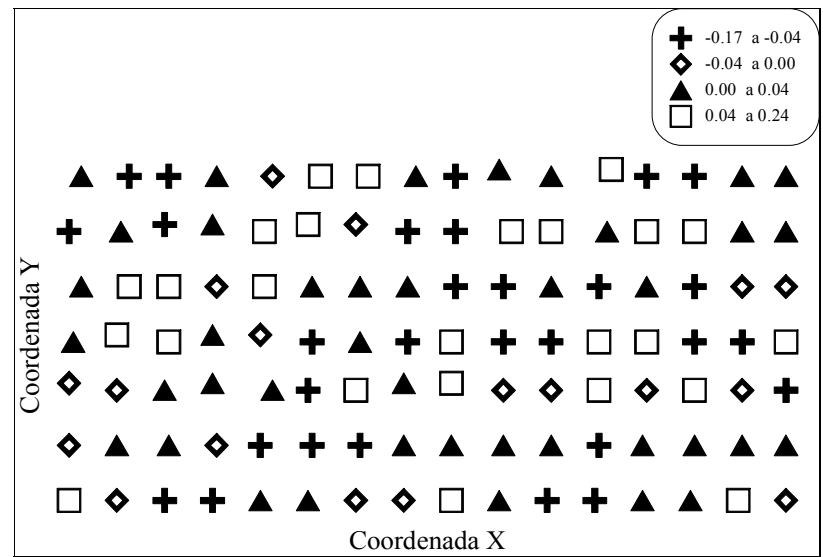

Figura 3. Distribuição espacial de densidade do solo (A). Distribuição espacial dos valores residuais de densidade do solo, após o refinamento pela mediana (B)

Comparando-se os semivariogramas escalonados de argila e areia, observa-se uma grande proximidade entre os dois modelos, o que também foi comprovado por Gonçalves (1997) significando que a diferença entre os modelos está na escala de variação dos dados, média e variância, sendo a estrutura de variação no espaço a mesma (Figura 4). Os semivariogramas 
apresentaram estrutura de dependência espacial, com efeito pepita de apenas $23 \%$ para o modelo de argila e areia $(\mathrm{SPh} 1)$ e $22 \%$ para silte (EXP 1). Esses resultados podem ser considerados de forte dependência, segundo escala utilizada por Cambardella et al. (1994).

A.

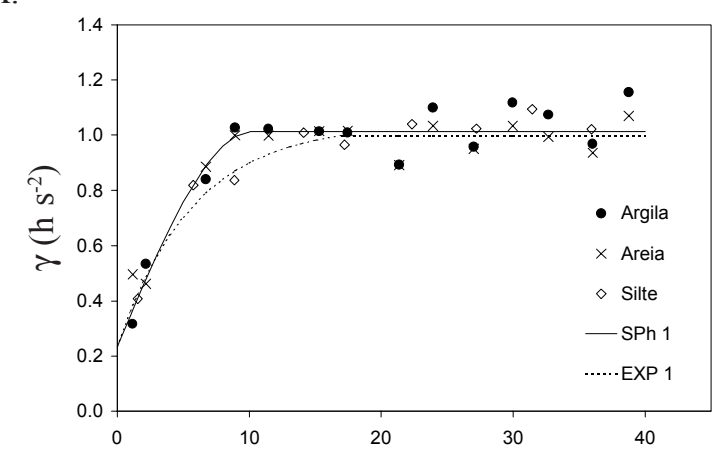

B.

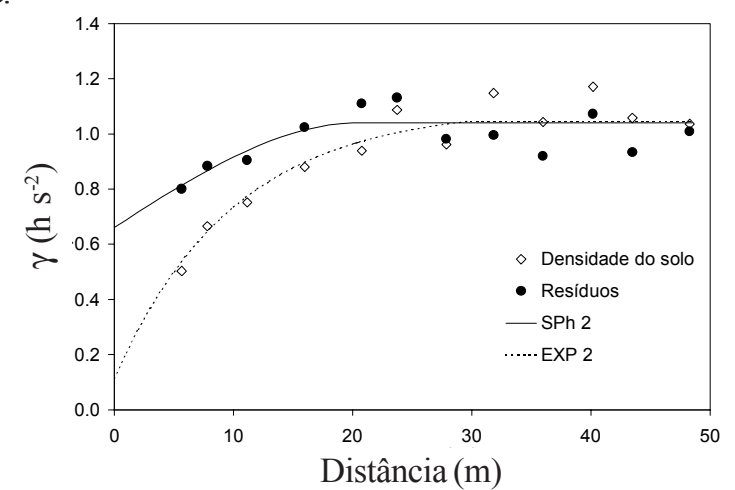

Figura 4. Semivariogramas escalonados para os teores de argila, areia e silte com respectivos modelos ajustados (A) e para os dados originais de densidade do solo e dos resíduos, com modelo ajustado (B)

Os semivariogramas escalonados para os dados originais de densidade do solo e dos resíduos do refinamento, revelaram que o efeito da variação de larga escala afetou os valores de semivariância e a técnica empregada na remoção da tendência foi eficiente e necessária para o bom desenvolvimento do trabalho. Comparativamente, percebe-se que para o semivariograma referente aos dados originais (EXP 2), a estrutura apresentou-se muito bem definida, com pequena contribuição do "nugget effect" (10\%) e possibilidade do ajuste de um modelo com alcance de até $30 \mathrm{~m}$, o que não foi possível com os resíduos .

Apesar do menor número de amostras coletadas para densidade do solo e estas amostras serem coletadas em espaçamento mínimo de $5 \mathrm{~m}$, foi possível a verificação da estrutura no semivariograma dos resíduos da densidade do solo. O modelo que melhor se ajustou aos valores de semivariância foi o esférico ( $\mathrm{SPh} 2) \mathrm{com}$ efeito pepita responsável por $63 \%$ da variação total dos dados e alcance de $21 \mathrm{~m}$ o que, segundo os critérios propostos por Cambradella et al. (1994) demonstra moderada dependência espacial. Os resultados dos coeficientes referentes aos modelos ajustados encontram-se na Tabela 3.

Para cada variável estudada, a interpolação pela "krigagem" foi realizada após a construção do melhor semivariograma, utilizando-se o número de vizinhos mais adequado, segundo os resultados da validação cruzada (Figura 5).
A.

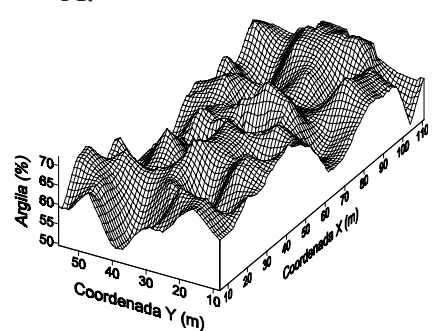

C.

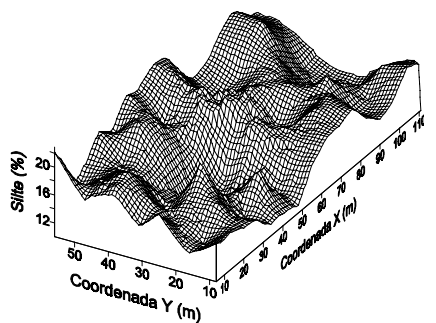

B.

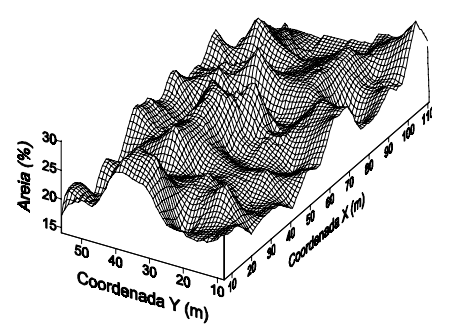

D.

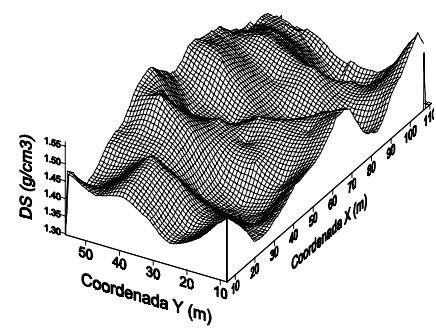

Figura 5. Distribuição espacial do conteúdo de argila (A), areia (B), silte (C) e da densidade do solo na área experimental (D)

Os mapas de argila e areia têm comportamento inverso, quando comparados entre si. A maior concentração de argila está na região inferior esquerda, que coincide com a que possui menor declividade, porém não se pode concluir que esta última foi determinante para as altas concentrações de argila na região, pois se verificam regiões em outros pontos e até na parte superior da área experimental, com altos teores dessa variável. É importante salientar que o processo de erosão existente é pouco significativo, visto que o sistema radicular da cultura é estável, por se tratar de cultura perene e, ainda, que a presença de ervas daninhas no centro das fileiras evita o maior arraste de partículas, além de não haver mobilização de terra decorrente do manejo da cultura, ou seja, o solo não fica descoberto.

Esta região de máxima concentração para argila deve ser analisada de maneira especial dentro da área, pois o conteúdo de silte também é muito baixo e, em grande parte, verifica-se que o conteúdo de areia atinge valores mínimos. Este comportamento certamente afeta a retenção de água na região. Apesar de possuir grande volume de água armazenado, pequena parte será disponível entre as tensões em que se realiza o manejo da irrigação localizada. Com relação ao teor de silte, o mapa também é inverso ao de argila, ou seja, baixos teores de argila correspondem a altos teores de areia e altos teores de silte, garantindo a característica intrínseca de um solo de textura "pesada".

Para a densidade do solo, o comportamento inicial verificado na Figura 4 foi ratificado, ou seja, maior concentração na parte superior da superfície, correspondendo à área central e de cota mais elevada da área experimental, decrescendo em direção à coordenada X. Notam-se, também, como já era esperado, valores altos para densidade do solo em zonas com maior conteúdo de areia. Este comportamento foi verificado, também, na região localizada próximo à coordenada $\mathrm{X}$, em pontos cujos valores para o conteúdo de areia foram mais altos. 


\section{Umidade do solo e água disponível}

A estatística descritiva para umidade do solo nas tensões estudadas pode ser analisada na Tabela 2, cujos valores indicam distribuição simétrica nas tensões estudadas, estando os valores de média e mediana próximos. Com relação à amplitude máxima das distribuições, verificou-se um comportamento de redução à medida que a tensão aumentou (Figura 6A). Tal comportamento também foi verificado por Moraes et al. (1993), trabalhando com 240 amostras de solo e oito níveis de tensão, em Terra Roxa Estruturada. Os autores atribuíram essa maior amplitude nas baixas tensões, à influência da estrutura na retenção da água.

Tabela 2. Resumo estatístico da umidade com base em massa nas tensões de 10,80 e $1500 \mathrm{kPa}$, percentagem de água disponível a 80 e $1.500 \mathrm{kPa}$ e correlação entre argila e a umidade $\left(\mathrm{R}^{2}\right)$

\begin{tabular}{lrrrrr}
\hline \multirow{2}{*}{ Parâmetros } & $\mathrm{U}_{\mathrm{CC}}$ & \multicolumn{1}{c}{$\mathrm{U}_{\mathrm{FT}}$} & \multicolumn{1}{c}{$\mathrm{U}_{\mathrm{PM}}$} & $\mathrm{D}_{\mathrm{LT}}$ & $\mathrm{D}_{\mathrm{PMI}}$ \\
\cline { 2 - 6 } & \multicolumn{5}{c}{$\%)$} \\
\hline $\mathrm{N}^{\mathrm{o}}$ de amostras & \multicolumn{1}{c}{112} & 112 & \multicolumn{1}{l}{112} & 112 & \multicolumn{1}{c}{112} \\
Média & 29,63 & 27,34 & 23,11 & 2,29 & 6,52 \\
Mediana & 29,59 & 27,36 & 23,46 & 2,04 & 6,06 \\
Mínimo & 23,81 & 22,19 & 17,71 & 1,11 & 4,03 \\
Máximo & 36,05 & 32,78 & 28,90 & 5,51 & 13,36 \\
Quartil inferior & 27,68 & 25,35 & 20,82 & 1,61 & 5,61 \\
Quartil superior & 31,29 & 29,25 & 25,39 & 2,71 & 7,20 \\
Amplitude & 12,24 & 10,59 & 11,19 & 1,11 & 1,60 \\
Desvio-Padrão & 2,73 & 2,57 & 2,72 & 0,94 & 1,56 \\
CV (\%) & 9,21 & 9,41 & 11,77 & 40,89 & 24,01 \\
Assimetria & 0,17 & $-0,01$ & $-0,14$ & 1,38 & 1,52 \\
Curtose & $-0,46$ & $-0,93$ & $-1,09$ & 1,79 & 3,44 \\
$\mathrm{R}^{2}$ & 0,58 & 0,55 & 0,50 & - & - \\
\hline
\end{tabular}

A.

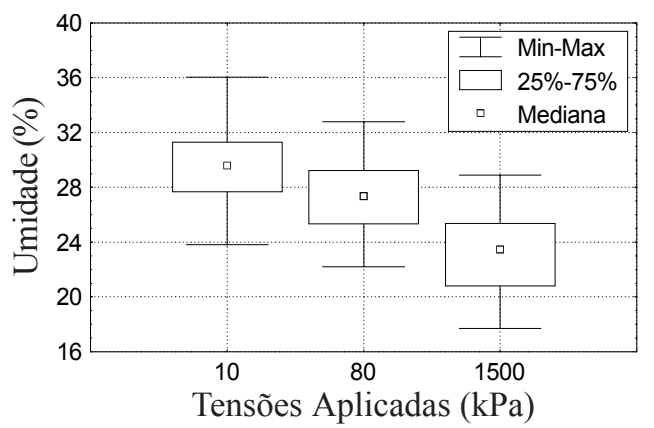

B.

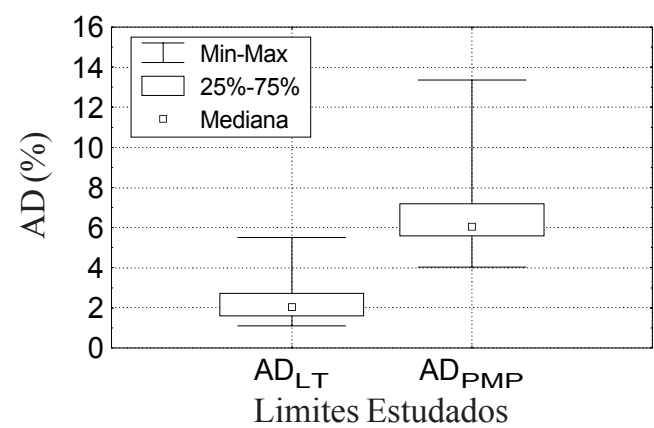

Figura 6. "Box-plot" para umidade com base em massa nas tensões correspondentes à capacidade de campo $\left(\mathrm{U}_{\mathrm{cc}}\right)$, limite de funcionamento do tensiômetro $\left(\mathrm{U}_{\mathrm{ff}}\right)$ e no ponto de murcha permanente $\left(U_{\mathrm{pmp}}\right)(\mathrm{A})$ e para água disponível no limite de funcionamento do tensiômetro $\left(\mathrm{AD}_{\mathrm{ft}} \%\right)$ e água disponível no ponto de murcha permanente $\left(\mathrm{AD}_{\mathrm{pmp}} \%\right)(\mathrm{B})$
Os valores para assimetria aproximaram-se de zero, indicando distribuições simétricas. As medidas de posição e o formato da distribuição indicam grande proximidade dos dados à distribuição normal, o que pôde ser confirmado com base nos resultados do teste de Kolmogorov-Smirnov, significativos em todos os níveis e em todas as tensões.

Para se analisar, em conjunto, a estrutura de dependência espacial da retenção de água, construíram-se semivariogramas experimentais escalonados em cada tensão estudada (Figura 7A). Foi possível o ajuste de um único modelo de semivariograma representando as umidades nas tensões estudadas (SPh 3) sendo a estrutura de variação no espaço, a mesma para todas as tensões. O modelo que permitiu o melhor ajuste aos semivariogramas, foi o esférico, com efeito pepita de apenas $16 \%$ do patamar com alcance de $13 \mathrm{~m}$. A partir do modelo ajustado, foi realizada a interpolação pelo processo da "krigagem", gerando mapas de superfície (Figura 8).

A.

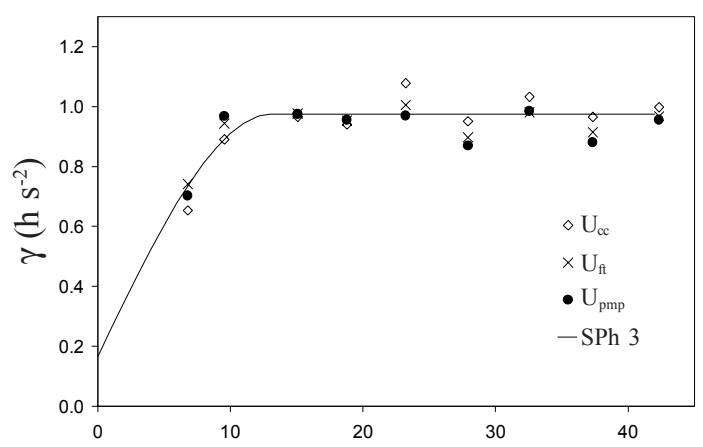

B.

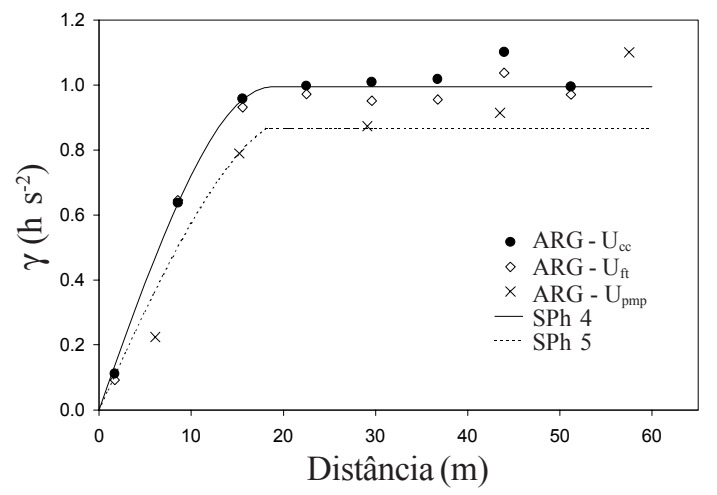

Figura 7. Semivariogramas escalonados da retenção de água $\left(\mathrm{U}_{\mathrm{cc}}, \mathrm{U}_{\mathrm{dt}} \mathrm{e} \mathrm{U}_{\mathrm{pmp}}\right)$ com modelo ajustado (A). Semivariogramas cruzados escalonados e modelos ajustados para argila e retenção de água no solo, nos níveis estudados (B)

A distribuição espacial da retenção de água nas tensões estudadas, mostrou grande semelhança com o mapa de argila, o que evidencia a existência de correlação espacial entre as variáveis, conforme avaliado por Gonçalves \& Folegatti (2000). Os coeficientes de determinação da correlação linear entre argila e retenção de água, indicam a existência de uma inter-relação entre as variáveis, na qual $50 \%$ da variação total da umidade são explicados pela variação do conteúdo de argila.

Os semivariogramas cruzados relacionando essas variáveis (Figura 8B) corroboram o que foi discutido, sendo possível o ajuste médio de um modelo para argila e retenção de água na $\mathrm{U}_{\mathrm{cc}}$ e $\mathrm{U}_{\mathrm{ft}}$, com efeito pepita nulo e alcance de $19 \mathrm{~m}$, além de um 

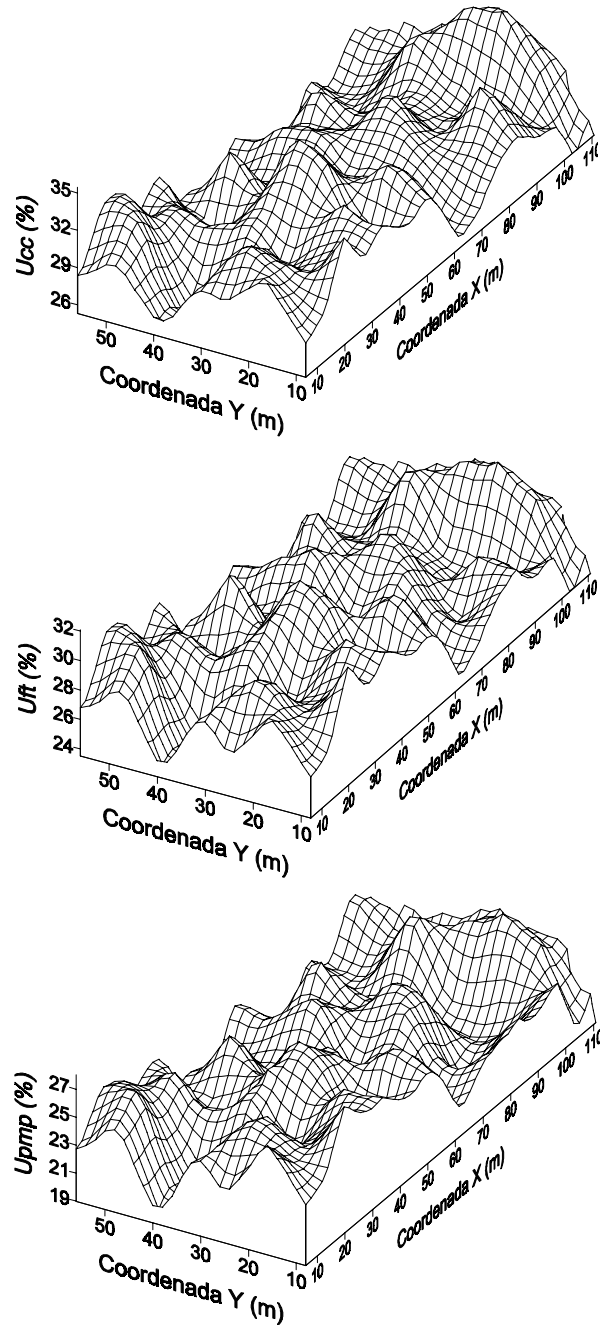

Figura 8. Distribuição espacial da umidade com base em massa nas tensões correspondentes à capacidade de campo $\left(\mathrm{U}_{\mathrm{cc}}\right)$, limite de funcionamento do tensiômetro $\left(\mathrm{U}_{\mathrm{ft}}\right)$ e no ponto de murcha permanente $\left(\mathrm{U}_{\mathrm{pmp}}\right)$

modelo para argila e $\mathrm{U}_{\text {pmp }}$, com efeito pepita nulo e alcance de $22 \mathrm{~m}$.

Comparando-se as superfícies de umidade com a de densidade do solo, verifica-se que, na região cuja densidade foi mais acentuada, os valores de umidade foram mais baixos, indicando algum problema de retenção de água ligado à compactação do solo, principalmente na tensão mais elevada. A estrutura do solo está ligada à retenção em tensões elevadas, sendo menos importante nas tensões mais baixas, quando a textura se torna mais relevante.

Os valores para $U_{c c}$ localizados entre 70 e $90 \mathrm{~m}$ da coordenada $Y$, que a princípio apareciam como zonas de umidade elevada aparecem, para a superfície de $\mathrm{U}_{\mathrm{ft}}$, com baixos valores de umidade. Esses dados indicam esta região como a que possui máximo armazenamento entre essas tensões. Pode-se verificar que tal região possui o teor mais elevado de areia, que pode refletir este resultado.

O resumo estatístico referente aos valores calculados de água disponível no limite de funcionamento do tensiômetro $\left(\mathrm{AD}_{\mathrm{ft}}\right)$ e no ponto de murcha permanente $\left(\mathrm{AD}_{\mathrm{pmp}}\right)$ indica a não normalidade da distribuição. Observa-se diferença expressiva entre a média e a mediana, indicando distribuição assimétrica, comprovada pelo valor da assimetria, bastante elevado, à semelhança do que ocorre com a curtose, que expressa distribuição leptocúrtica, indicando a presença de pontos afastados do centro da distribuição. Na Figura 6B pode-se verificar esse comportamento irregular da distribuição nos gráficos de "box-plot", em que pontos se afastam do centro da distribuição. Assim como os resultados anteriores, o teste de Kolmogorov-Smirnov negou a hipótese de normalidade dos dados. Os CV para AD indicaram média variabilidade e mostram que esta variável, apesar de ser derivada da retenção de água, não apresentou as características das variáveis que lhe deram origem, que apresentaram distribuição normal e baixa variabilidade. Resultados semelhantes foram encontrados por Moraes \& Libardi (1993). No presente estudo, pode-se verificar maior variabilidade para $\mathrm{AD}_{\mathrm{ft}}$, que reflete a modificação da estrutura do solo, devido à compactação em parte da área estudada. O manejo de irrigação localizada via tensiometria, ocorre nessa faixa de estudo e a variabilidade mais elevada tende a dificultar a interpretação dos resultados obtidos em situações como esta. A Figura 9 apresenta os mapas de contorno para $\mathrm{AD}_{\mathrm{ft}}$ e $\mathrm{AD}_{\mathrm{pmp}}$, resultantes dos mapas de umidade.

A.

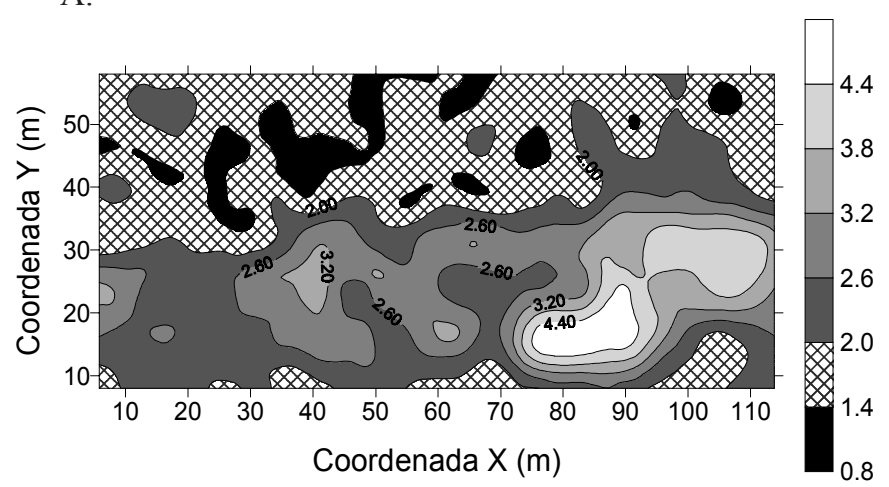

B.

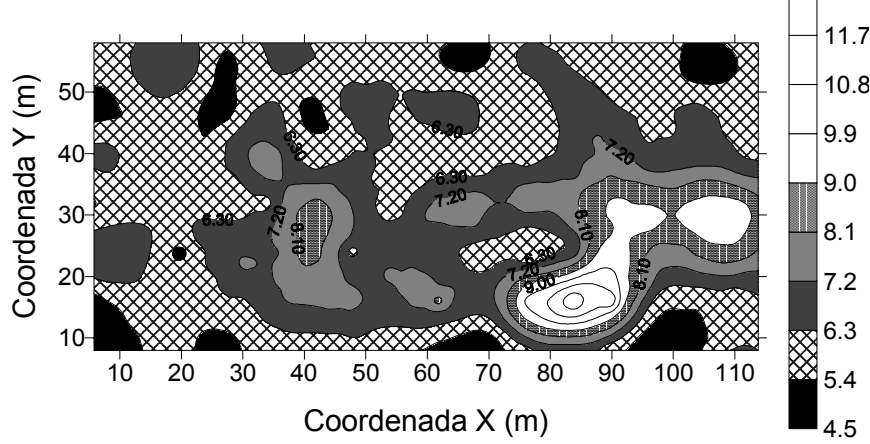

Figura 9. Mapas de contorno para água disponível no limite de funcionamento do tensiômetro $\left(\mathrm{AD}_{\mathrm{ft}} \%\right)(\mathrm{A})$ e água disponível no ponto de murcha permanente $\left(\mathrm{AD}_{\mathrm{pmp}} \%\right)(\mathrm{B})$

Nota-se maior disponibilidade de água na parte baixa da superfície, em direção à coordenada $\mathrm{X}$ e, para a região superior do terreno, observam-se baixos valores de água armazenada. A argila teve influência nos altos valores de disponibilidade de água, que coincidem com os locais onde o seu teor foi mais elevado, porém a existência de outros fatores, como a possível compactação do solo na parte superior da área experimental, pode ter influenciado nos baixos valores de armazenamento em 
resposta à modificação na estrutura do solo, resultando em uma estrutura espacial da disponibilidade de água diferente das demais e que não pode ser explicada somente por uma variável.

Irrigação com alta freqüência poderia minimizar o problema da alta variabilidade da $\mathrm{AD}$, repondo-se apenas o que foi evapotranspirado pela cultura, estimada por um método agrometeorológico, mas só seria eficiente quando em baixa variabilidade do vigor entre plantas, condição difícil de ocorrer em culturas perenes que, ao longo do tempo de cultivo, respondem diferentemente ao manejo agronômico adotado e à variabilidade do solo. Parece ser mais prudente, quando se busca a eficiência do uso de água, realizar antes a subdivisão da área em zonas homogêneas quanto ao armazenamento para, posteriormente, se definir um manejo de irrigação diferenciado em cada sub-região. A utilização da descrição espacial das variáveis mais importantes no manejo de irrigação auxilia um manejo mais racional de água na área experimental e o controle local pode ser realizado após a subdivisão da área, em zonas mais homogêneas.

Tabela 3. Coeficientes dos modelos ajustados aos semivariogramas experimentais: efeito pepita $\left(\mathrm{C}_{\mathrm{o}}\right)$, "sill" $(\mathrm{C})$, patamar $\left(\mathrm{C}+\mathrm{C}_{\mathrm{o}}\right) \mathrm{e}$ alcance (a)

\begin{tabular}{lllccc}
\hline Modelo & Co & C & $\mathrm{C}+\mathrm{C}_{\mathrm{o}}$ & $\mathrm{a}(\mathrm{m})$ & $\mathrm{C}_{\mathrm{o}} /\left(\mathrm{C}_{\mathrm{o}}+\mathrm{C}\right)$ \\
\hline SPh 1 & 0,23 & 0,78 & 1,01 & 10 & 0,23 \\
SPh 2 & 0,66 & 0,38 & 1,04 & 21 & 0,63 \\
SPh 3 & 0,16 & 0,81 & 0,97 & 13 & 0,16 \\
SPh 4 & 0 & 0,99 & 0,99 & 19 & 0 \\
SPh 5 & 0 & 0,91 & 0,91 & 22 & 0 \\
EXP 1 & 0,23 & 0,81 & 1,04 & 17 & 0,22 \\
EXP 2 & 0,11 & 0,98 & 1,09 & 30 & 0,10 \\
\hline
\end{tabular}

\section{CONCLUSÕES}

1. O coeficiente de variação $(\mathrm{CV})$ permitiu caracterizar-se as variáveis $U_{c c}, U_{f t}, U_{p m p}$ e argila, como de baixa variação. Os valores de $\mathrm{CV}$ foram crescentes nesta ordem. Para as variáveis areia, silte, $\mathrm{AD}_{\mathrm{pmp}}$ e $\mathrm{AD}_{\mathrm{ft}}$, os valores de $\mathrm{CV}$, crescentes nesta ordem, permitiram caracterizá-las como de média variação.

2. As variáveis estudadas apresentaram estrutura de dependência espacial, o que permitiu o seu mapeamento, utilizando-se técnicas geoestatísticas.

3. Há estreita correlação espacial entre conteúdo de argila e retenção de água no solo, descrita pelos semivariogramas cruzados para essas propriedades.

4. O mapeamento das variáveis estudadas permite o estabelecimento de subáreas mais homogêneas, nas quais se pode realizar, de maneira mais eficiente, o manejo de água.

\section{LITERATURA CITADA}

Burden, D.S.; Selim, H.M. Correlation of spatially variable soil water retention for a surface soil. Soil Science, Baltimore, v.148, n.6, p.436-447, 1989.

Cambardella, C.A.; Moorman, T.B.; Novak, J.M.; Parkin, T.B.; Karlen, D.L.; Turco, R.F.; Konopka, A.E. Field-scale variability of soil properties in Central Iowa Soils. Soil Science Society of America Journal, Madison, v.58, p.1501-1511, 1994.
Castrignanò, A.; Stelluti, M. Fractal geometry and geostatistics for describing the field variability of soil aggregation. Journal of Agricultural Engineering Research, London, v.73, p.13-18, 1999.

Gonçalves, A.C.A. Variabilidade espacial de propriedades físicas do solo para fins de manejo da irrigação. Piracicaba: ESALQ/USP, 1997, 118p. Tese Doutorado

Gonçalves, A.C.A.; Folegatti, M.V. Correlação espacial entre retenção de água e textura do solo, para fins de manejo de irrigação. In: Congresso Brasileiro de Engenharia Agrícola, 29, Fortaleza: Anais... 2000. CD-Rom

Hamlett, J.M.; Horton, R.; Cressie, N.A.C. Resistant and exploratory techniques for use in semivariogram analyses. Soil Science Society of America Journal, Madison, v.50, p.868-875, 1986.

Journel, A.G.; Huijbregts, Ch. J. Mining geostatistics. London: Academic Press, 1978. 600p.

Libardi, P.L.; Manfron, P.A.; Moraes, S.O. de; Tuon, R.L. Variabilidade da umidade gravimétrica de um solo hidromórfico. Revista Brasileira de Ciência do Solo, Viçosa, v.20, p.1-12, 1996.

McBratney, A.B.; Webster, R. Choosing functions for semi-variograms of soil properties and fitting them to sampling estimates. Journal of Soil Science, Oxford, v.37, p.617-639, 1986.

Moraes, S.O.; Libardi, P.L. Variabilidade da água disponível de uma Terra Roxa Estruturada Latossólica. Scientia Agricola, Piracicaba, v.50, n.3, p.404-412, 1993.

Moraes, S.O.; Libardi, P.L.; Reichardt, K. Heterogeneidade dos pontos experimentais de curva de retenção da água no solo. Scientia Agricola, Piracicaba, v.50, n.3, p.393-403, 1993.

Oliveira, J.B. Variação de características morfológicas, físicas e mineralógicas em duas áreas de oxossolo. Piracicaba: ESALQ/USP, 1973. 199p. Tese Doutorado

Paz, A.; Taboada, M.T.; Gómez, M.J. Spatial variability in topsoil micronutrient contents in a one-hectare cropland plot. Communications in Soil Science and Plant Analysis, New York, v.27, n.3/4, p.479-503, 1996.

Souza, L. da S.; Cogo, N.P.; Vieira, S.R. Variabilidade de propriedades físicas e químicas em um pomar cítrico. Revista Brasileira de Ciência do Solo, Viçosa, v.21, p-367-372, 1997.

Trangmar, B.B.; Yost, R.S.; Uehara, G. Application of geostatistics to spatial studies of soil properties. Advances in Agronomy, New York, v.38, p.45-94, 1985.

Vauclin, M.; Vieira, S.R.; Vachaud, G.; Nielsen, D.R. The use of cokriging with limited soil observations. Soil Science Society of America Journal, Madison, v.47, n.2, p175-184, 1983.

Vieira, S.R.; Maria, I.C. de. Delineamento experimental e análise estatística na pesquisa de conservação do solo. In: Reunión sobre metodologia para investigatión en manejo de suelos, Dialogo. Montevideo: IICA, 1995. p.3-11.

Vieira, S.R.; Tillotson, P.M.; Biggar, J.W.; Nielsen, D.R. Scaling of semivariograms and the Kriging estimation of field-measured properties. Revista Brasileira de Ciência do Solo, Viçosa, v.21, p. 525-533, 1997.

Warrick, A.W.; Nielsen, D.R. Spatial variability of soil physical properties in the field. In: Hillel, D. (ed.). Application of soil physics. New York: Academic Press, 1980, p.319-344. 